\title{
Missense mutations of $M L H 1$ and $M S H 2$ genes detected in patients with gastrointestinal cancer are associated with exonic splicing enhancers and silencers
}

\author{
MING ZHU ${ }^{1,2}$, HUI-MEI CHEN ${ }^{1}$ and YA-PING WANG ${ }^{1}$ \\ ${ }^{1}$ Jiangsu Key Laboratory of Molecular Medicine, Nanjing University School of Medicine, Nanjing 210093; \\ ${ }^{2}$ Department of Molecular Biology, Jiangsu Institute of Cancer Research, Nanjing 210009, P.R. China
}

Received November 23, 2012; Accepted February 18, 2013

DOI: $10.3892 / \mathrm{ol} .2013 .1243$

\begin{abstract}
The $M L H 1$ and $M S H 2$ genes in DNA mismatch repair are important in the pathogenesis of gastrointestinal cancer. Recent studies of normal and alternative splicing suggest that the deleterious effects of missense mutations may in fact be splicing-related when they are located in exonic splicing enhancers (ESEs) or exonic splicing silencers (ESSs). In this study, we used ESE-finder and FAS-ESS software to analyze the potential ESE/ESS motifs of the 114 missense mutations detected in the two genes in East Asian gastrointestinal cancer patients. In addition, we used the SIFT tool to functionally analyze these mutations. The amount of the ESE losses (68) was $51.1 \%$ higher than the ESE gains (45) of all the mutations. However, the amount of the ESS gains (27) was $107.7 \%$ higher than the ESS losses (13). In total, 56 (49.1\%) mutations possessed a potential exonic splicing regulator (ESR) error. Eighty-one mutations (71.1\%) were predicted to be deleterious with a lower tolerance index as detected by the Sorting Intolerant from Tolerant (SIFT) tool. Among these, 38 $(33.3 \%)$ mutations were predicted to be functionally deleterious and possess one potential ESR error, while 18 (15.8\%) mutations were predicted to be functionally deleterious and exhibit two potential ESR errors. These may be more likely to affect exon splicing. Our results indicated that there is a strong correlation between missense mutations in $M L H 1$ and MSH2 genes detected in East Asian gastrointestinal cancer patients and ESR motifs. In order to correctly understand the molecular nature of mutations, splicing patterns should be compared between wild-type and mutant samples.
\end{abstract}

Correspondence to: Dr Ya-Ping Wang, Jiangsu Key Laboratory of Molecular Medicine, Nanjing University School of Medicine, 22 Hankou Road, Nanjing 210093, P.R. China

E-mail: wangyap@nju.edu.cn

Key words: gastrointestinal cancer, missense mutation, exonic splicing enhancer, exonic splicing silencer, $M S H 2, M L H 1$

\section{Introduction}

The incidence and mortality rates of gastric cancer and colorectal cancer are among the highest among malignant tumors in East Asia. Germline mutations of mismatch repair (MMR) genes are responsible for the majority of hereditary nonpolyposis colorectal cancer (HNPCC) cases. The MMR genes MSH2 (OMIM No. 609309) and MLH1 (OMIM No. 120436) are considered to be the two major genes implicated in HNPCC $(1,2)$. Carriers of germline mutations of MSH2 and $M L H 1$ genes have a 4-fold greater risk of gastric cancer compared with normal individuals, as well as a high risk of colorectal cancer. These two genes are associated with gastrointestinal cancer susceptibility.

Missense mutations are among the most common types of mutations underlying inherited human diseases. The deleterious effects of missense mutations are usually attributed to their effects on the structure or function of a protein. The assumption may be misleading, as the mutations that affect the sequences that are important for splicing modulation are likely to have a profound effect on the translated product. It has become increasingly clear that exonic point mutations located outside the splice sites may affect pre-mRNA splicing and thereby cause disease $(3,4)$. Correct pre-mRNA splicing not only requires that the splice site sequences are present at the exon-intron borders, but is also critically dependent on additional intronic and exonic regulatory sequences (5). Those present in exons and with the capacity of enhancing splicing are called exonic splicing enhancers (ESEs) and those with the capacity of inhibiting the splicing are the exonic splicing silencers (ESSs). Generally, these classes of elements are called exonic splicing regulators (ESRs). Consequently, mutations located in ESE or ESS elements may affect splicing. The significance and prevalence of this phenomenon may have been significantly underestimated, as the majority of studies of disease-related genes are limited to the analysis of genomic DNA.

The majority of enhancer sequences within exons have been found to bind members of the serine/arginine-rich (SR) protein family, while many silencing elements are bound by members of the heterogeneous nuclear ribonuclearprotein (hnRNP) family (6). ESEs are discrete, degenerate motifs of 
6-8 nts located inside exons $(7,8)$. The study of normal splicing suggests that the majority of exons contain at least one functional ESE site (7,9). ESE-bound SR proteins promote exon definition by directly recruiting and stabilizing the splicing machinery through protein-protein interactions (10), and/or by antagonizing the function of nearby silencer elements (11). The cores of ESSs are considered to be relatively short (6-10 nts). ESS-bound hnRNPs are proposed to mediate silencing through direct antagonism of the splicing machinery or by direct competition for overlapping enhancer binding sites. The intrinsic strength by which the splice sites are recognized by the spliceosome, as well as the antagonistic dynamics of proteins binding ESEs and ESSs, control a large proportion of exon recognition and alternative splicing. Therefore, exonic splicing regulatory sequences are now increasingly recognized as a major target and a common mechanism for disease-causing mutations leading to exon skipping in functionally diverse genes.

In this study, we used ESE-finder $(12,13)$ and FAS-ESS (14) software to analyze the missense mutations of $\mathrm{MSH} 2$ and MLH1 genes detected in East Asian gastrointestinal cancer patients, and to assess whether these mutations hit the predicted ESE/ESS motifs and affected gene splicing.

\section{Materials and methods}

Subjects. A total of 114 missense mutations, 52 of $M S H 2$ and 62 of $M L H 1$, detected in the gastrointestinal cancer patients, were serially collected for this study from published East Asian literature (15-44) (Table I). The majority of the investigated mutations were exclusively reported in East Asia (China, Japan and Korea), and some of the mutations were detected in different ethnicities. The study was approved by the Ethics Committee of Nanjing University, Nanjing, China.

Potential ESE motif analysis. To identify the ESE motifs that were recognized by individual SR proteins, a PCR-based systematic evolution of ligands by exponential enrichment (SELEX) was used. During this approach, a natural splicing enhancer in a minigene was replaced by short, random sequences derived from an oligonucleotide library. The generated pool of minigenes was transfected into cultured cells, and spliced mRNAs were amplified by RT-PCR and sequenced (7). On the basis of the frequencies of the individual nucleotides at each position, a score matrix for each nucleotide in each position was calculated. This score matrix may be used to predict SR protein-specific ESEs (ESE-finder) $(12,13)$.

We analyzed wild-type or mutant exon sequences from MLH1 and MSH2 genes in Table I with ESE-finder software using SR protein score matrices and threshold values, essentially as described previously (ESE-finder: http://rulai. cshl.edu/tools/ESE/) (12). Sequence motifs for the same or different SR proteins may overlap. We considered only the wild type or mutant sequence motifs with scores greater than or equal to the value of the threshold for the corresponding SR protein. The threshold values were as follows: SF2/ASF (IgM-BRCA1) heptamer motif, 1.867; SC35 octamer motif, 2.383; SRp40 heptamer motif, 2.670 and SRp55 hexamer motif, 2.676.
Potential ESS motif analysis. To systematically identify ESS motifs, an in vivo splicing reporter system was developed to screen a library of random decanucleotides. The resulting library was transfected into cultured human 293 cells, and stably transfected cells were combined and sorted for GFP-expressing cells by fluorescence activated cell sorting (FACS) analysis. The fluorescence-activated screen for exonic splicing silencers (FAS-ESS, or FAS for short) yielded 176 ESS hexamers (FAS-hex2 set) (14).

We analyzed wild-type or mutant exon sequences from MLH1 and MSH 2 genes in Table I with FAS-ESS software using a FAS-hex2 set (176 ESS hexamers), essentially as described previously (FAS-ESS: http://genes.mit.edu/ fas-ess/) (14).

SIFT analysis. Sorting Intolerant from Tolerant (SIFT) tool (accessible at http://sift.jcvi.org/) was applied to detect deleterious missense mutations $(45,46)$. SIFT compiles a dataset of functionally linked protein sequences by searching the protein database using a PSI-BLAST algorithm. Subsequently, it builds an alignment from the homologous sequences with the query sequence and scans all positions in the alignment, as well as calculating the probabilities for amino acids at that position. The substitution at each position with normalized probabilities of a tolerance index or SIFT score of $<0.05$ are predicted to be deleterious or intolerant, while those $\geq 0.05$ are predicted to be tolerant (45). In this study, reference sequence (RefSeq) ID or GenInfo Identifier (GI) number and substitutions were provided as inputs to the SIFT blink program (46). A total of 52 missense mutations in the MSH2 gene (GI: 4557761) and 62 in the $M L H 1$ gene (GI, 463989) were analyzed for identification of deleterious variants.

\section{Results}

Potential ESE/ESS analysis of the mutations in MLH1 and MSH2 genes. We analyzed wild-type or mutant exon sequences from $M L H 1$ and $M S H 2$ genes in Table I using SR protein score matrices and threshold values, essentially as described. Potential ESE motifs found in the mutations in the two genes are listed in Table I (Fig. 1). Some of the mutations may load in different potential ESE motifs. Of the 114 mutations analyzed, 47 (41.2\%) mutations resulted in 68 ESE motif scores (24 SF2/ASF [IgM-BRCA1], 13 SC35, 19 SRp40 and 12 SRp55) being eliminated. However, 38 (33.3\%) mutations created 45 ESE motif scores (12 SF2/ASF [IgM-BRCA1], 8 SC35, 14 SRp40 and 11 SRp55).

We analyzed wild-type or mutant exon sequences from MLH1 and MSH2 genes in Table I with FAS-ESS using the FAS-hex2 set (176 ESS hexamers), essentially as described previously. Potential ESS motifs found in the mutations in the two genes are listed in Table I (Fig. 2). Of the 114 mutations assessed, 9 (7.9\%) mutations resulted in 13 ESS motif scores being eliminated. However, 17 (14.9\%) mutations created 27 ESS motif scores.

Eliminating the potential ESE motif and creating the potential ESS motif have the same effect on exon exclusion. We named these mutations as potential ESR error mutations. In total, 56 (49.1\%) mutations possessed a potential ESR error (Table II). 


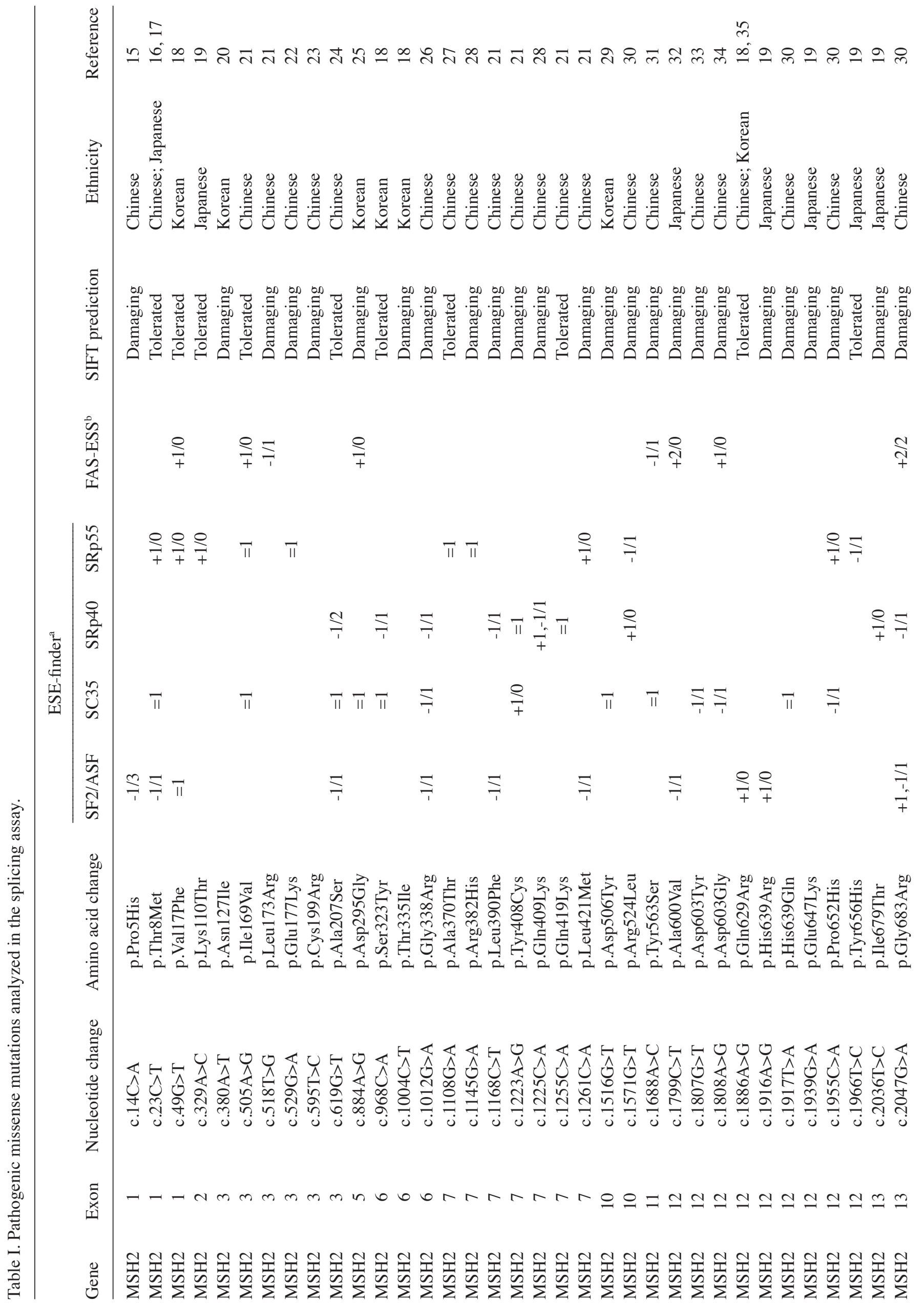









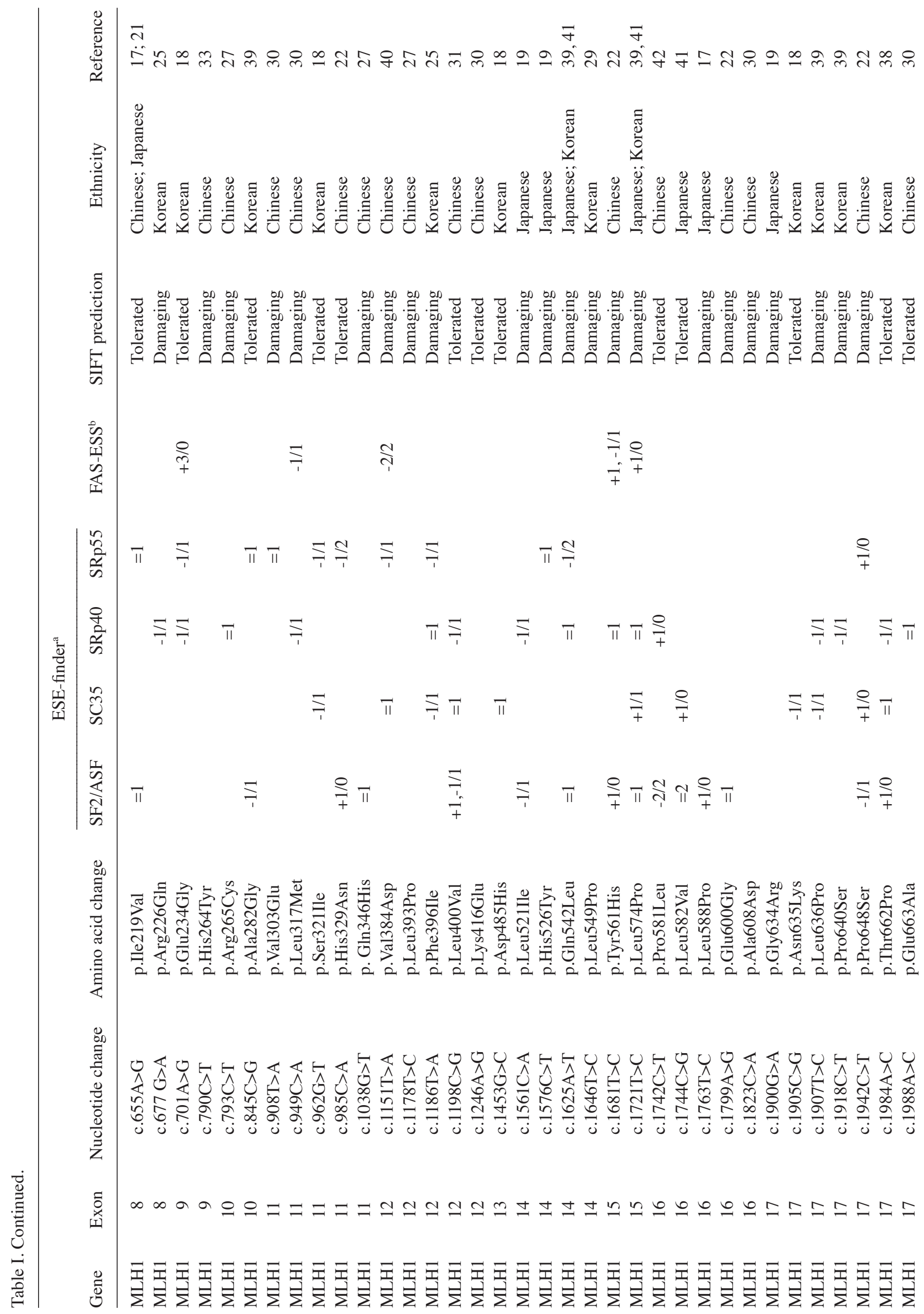



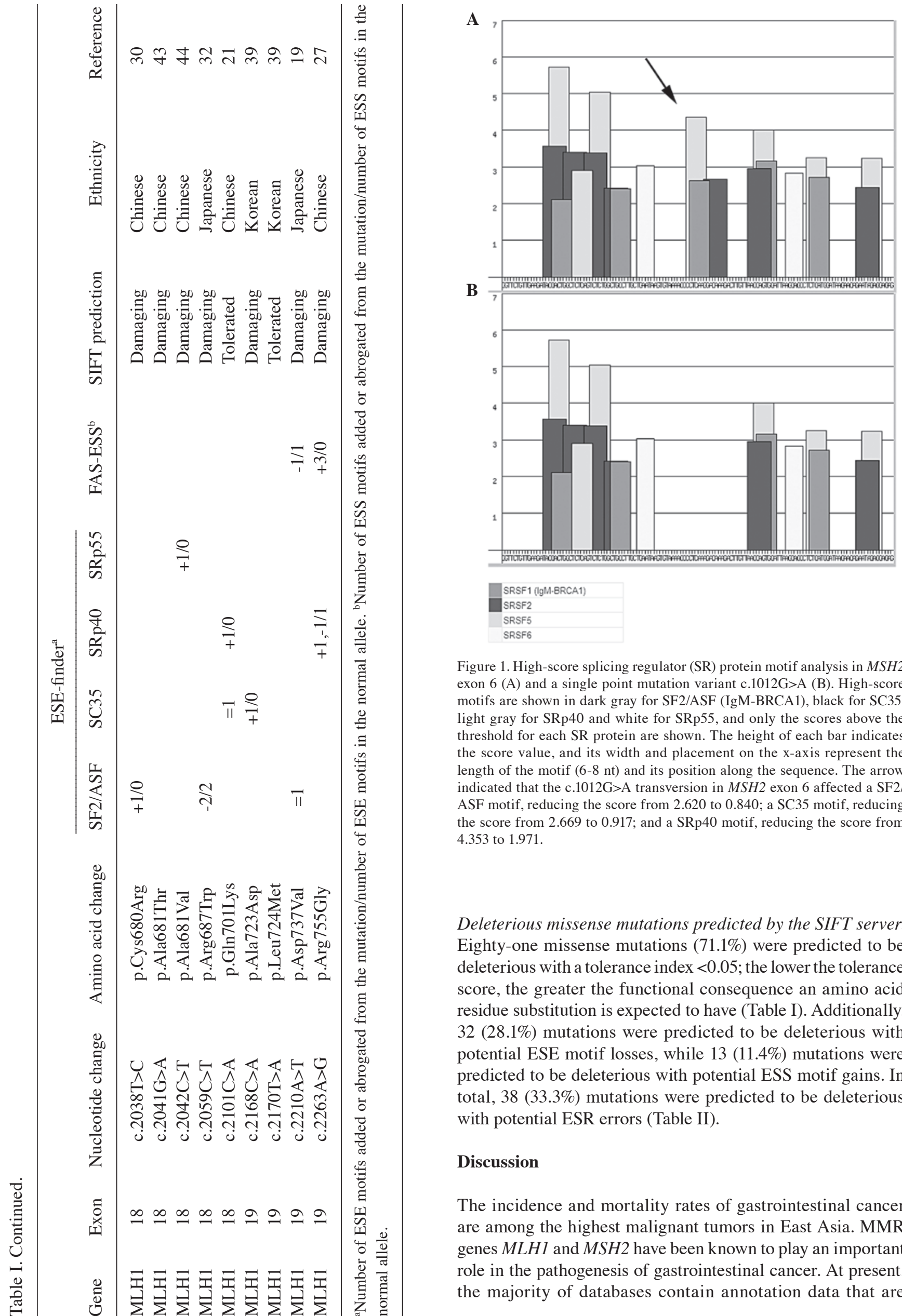

Figure 1. High-score splicing regulator (SR) protein motif analysis in $\mathrm{MSH} 2$ exon 6 (A) and a single point mutation variant c.1012G $>$ A (B). High-score motifs are shown in dark gray for SF2/ASF (IgM-BRCA1), black for SC35, light gray for SRp40 and white for SRp55, and only the scores above the threshold for each SR protein are shown. The height of each bar indicates the score value, and its width and placement on the $\mathrm{x}$-axis represent the length of the motif (6-8 nt) and its position along the sequence. The arrow indicated that the c.1012G $>$ A transversion in $M S H 2$ exon 6 affected a SF2 ASF motif, reducing the score from 2.620 to 0.840 ; a SC35 motif, reducing the score from 2.669 to 0.917 ; and a SRp40 motif, reducing the score from 4.353 to 1.971 .

Deleterious missense mutations predicted by the SIFT server. Eighty-one missense mutations $(71.1 \%)$ were predicted to be deleterious with a tolerance index $<0.05$; the lower the tolerance score, the greater the functional consequence an amino acid residue substitution is expected to have (Table I). Additionally, $32(28.1 \%)$ mutations were predicted to be deleterious with potential ESE motif losses, while 13 (11.4\%) mutations were predicted to be deleterious with potential ESS motif gains. In total, 38 (33.3\%) mutations were predicted to be deleterious with potential ESR errors (Table II).

\section{Discussion}

The incidence and mortality rates of gastrointestinal cancer are among the highest malignant tumors in East Asia. MMR genes $M L H 1$ and $M S H 2$ have been known to play an important role in the pathogenesis of gastrointestinal cancer. At present, the majority of databases contain annotation data that are 
Table II. Potential ESR errors detected in the mutations of the two genes.

\begin{tabular}{lccccc}
\hline & Total mutations & ESE eliminated & ESS created & ESR error & Two ESRs error \\
\hline Total mutations & $114(100.0 \%)$ & $47(41.2 \%)$ & $17(14.9 \%)$ & $56(49.1 \%)$ & $25(21.9 \%)$ \\
SIFT deleterious & $81(71.1 \%)$ & $32(28.1 \%)$ & $13(11.4 \%)$ & $38(33.3 \%)$ & $18(15.8 \%)$ \\
\hline
\end{tabular}

ESR error; mutations that have lost one ESE motif or gained one ESS motif. Two ESRs error; mutations that have eliminated at least two potential ESE motifs, or created at least two potential ESS motifs, or eliminated one or more potential ESE motifs and created one or more potential ESS motifs. ESR, exonic splicing regulator; ESE, exonic splicing enhancer; ESS, exonic splicing silencer.

A

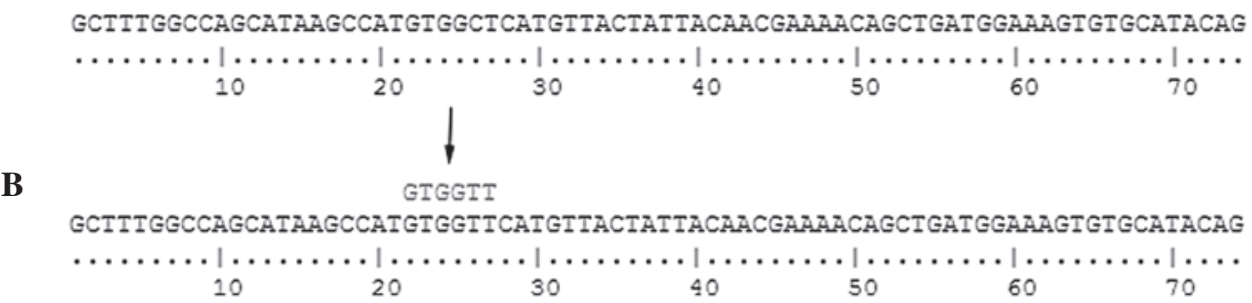

Figure 2. Fluorescence-activated screen for exonic splicing silencers (FAS-ESS) analysis of $M L H 1$ exon 4 (A) and a single point mutation variant c.332C $>$ T (B). The arrow indicates that the c.332C $>\mathrm{T}$ transversion in $M L H 1$ exon 4 created a new ESS motif.

primarily or exclusively derived from genomic DNA analysis, and the effect of a mutation on the mRNA or on the encoded protein is usually predicted from the primary sequence, rather than by experimentally determining the mRNA expression and splicing patterns. Therefore, the majority of reported disease-associated alleles of these genes are small insertions, deletions or splice-site mutations that result in protein truncation. Thus, only a small number of amino acid substitutions in either gene have been described as deleterious missense mutations, yet a very large number of different unclassified variant alleles are routinely encountered in clinical and research laboratories. It is therefore necessary to functionally define these unclassified variants as deleterious alleles, low-penetrance alleles or benign polymorphisms.

In this study, we selected 114 missense mutations of $M S H 2$ and $M L H 1$ genes detected from East Asian gastrointestinal cancer patients in published studies. The ethnic group included Chinese, Japanese and Korean individuals. The missense mutations contribute to certain forms of cancer susceptibility in East Asian populations, but it was unclear whether these were the definite pathogenic mutations in gastrointestinal cancer.

The consequences of splicing unclassified variants found in the $M L H 1$ or the $M S H 2$ genes may be studied directly at the patient RNA level. However, the number of variants that may be tested for splicing alterations using patient RNA is limited by the difficulty of obtaining blood samples suitable for RNA extraction. The bioinformatic tools, the ESE-finder and FAS-ESS, may enable prediction of the splicing defect of the mutations. These tools have already been used successfully to predict ESEs/ESSs and their disruption in a variety of genes, including ACF, BRCA1, BRCA2, FBN1, IGF1, PDHA1, SMN1, SMN2, TNFRSF5, CFTR, MlH1, MSH2, Tp53, MCAD and others (3,7,47-52). Auclair et al conducted a systematic
RNA screening of a series of 60 western patients who carried unrelated exonic or intronic mutations in the $\mathrm{MLH1}$ or $\mathrm{MSH} 2$ genes (53). In addition, it was found that the potential correlation between aberrant splicing and prediction of ESE by the ESE-finder demonstrated a sensitivity of $80 \%$ and a specificity of $42 \%$.

Under the conditions of the null hypothesis, there is no correlation between ESEs and mutations; the amount of ESE motif scores eliminated or created should be equal. However, in the present study, the amount of ESE losses (68) was 51.1\% higher than ESE gains (45). This suggested that the mutations loaded in the potential ESE motifs were more likely to eliminate the ESE motif score, and that they affected gene splicing. Additionally, under the conditions of the null hypothesis, there is no correlation between ESSs and mutations; the amount of ESS motif scores eliminated or created should be equal. Conversely, in the present study, the amount of ESS gains (27) was $107.7 \%$ higher than the amount of ESS losses (13). This suggested that the mutations were more likely to create the ESS motif score and that they affected gene splicing, indicating that there is a strong association between missense mutations in $\mathrm{MLH} 1$ and $\mathrm{MSH} 2$ genes and ESE/ESS motifs. Some of the mutations should be splicing-related deleterious alleles.

As an upper limit for the estimate of the proportion of ESR-related mutations, we suggest that 56 (49.1\%) mutations, which have lost ESE or gained ESS motifs, were deleterious for the reason that they disturbed functional splicing enhancers or or created functional splicing silencers, respectively. This approach was likely to overestimate the proportion of ESR-related pathogenic mutations. This is due to the fact that not all ESR motifs are true functional ESRs, and not all nucleotide substitutions in functional ESRs disturb their function. 
According to previous studies, no extensive functional analysis was available for these mutations. We used the SIFT tool to functionally analyze the missense mutations. SIFT is a program that predicts the effect of amino acid substitutions on protein function, on the basis of sequence conservation during evolution and the nature of the amino acids substituted in a gene of interest. In total, 81 missense mutations (71.1\%) were predicted to be deleterious with a tolerance index $<0.05$. Among them, 38 (33.3\%) mutations were predicted to be deleterious and have at least one potential ESR error. Some of these may be pathogenic with exon exclusion.

Eliminating and creating the potential ESE motif has the same effect on exon exclusion. One mutation may eliminate one or more potential ESE motifs. The greater the number of potential ESE motifs eliminated, the more likely the mutation was to affect the ESE motifs. However, one mutation may create one or more potential ESS motifs. The greater the number of potential ESS motifs created, the more likely the mutation was to affect the ESS motifs. In total, 25 (21.9\%) mutations eliminated at least two potential ESE motifs, or created at least two potential ESS motifs, or eliminated one or more potential ESE motifs and created one or more potential ESS motifs. These may be more likely to affect exon splicing. Among these, 18 (15.8\%) mutations, c.1012G $>$ A, c. $1168 \mathrm{C}>\mathrm{T}$, c. $1799 \mathrm{C}>\mathrm{T}$, c. $1808 \mathrm{~A}>\mathrm{G}$, c. $2047 \mathrm{G}>\mathrm{A}$, c. $2064 \mathrm{G}>\mathrm{A}$, c. $2108 \mathrm{C}>\mathrm{A}$, c. $2128 \mathrm{G}>\mathrm{T}$, c. $2141 \mathrm{C}>\mathrm{T}$ in $M S H 2$; c. $242 \mathrm{C}>\mathrm{T}$, c. $318 \mathrm{C}>\mathrm{G}$, с. $327 \mathrm{~T}>\mathrm{G}$, с. $332 \mathrm{C}>\mathrm{T}$, с. $1186 \mathrm{~T}>\mathrm{A}$, с. $1561 \mathrm{C}>\mathrm{A}$, c. $1907 \mathrm{~T}>\mathrm{C}$, c. $2059 \mathrm{C}>\mathrm{T}, \mathrm{c} .2263 \mathrm{~A}>\mathrm{G}$ in $M L H 1$, were predicted to be deleterious in the SIFT analysis. These were the mutations that most likely affected exon splicing, and were denoted as ESR-relevant mutations. We proposed that some of these disrupted functional ESEs or created functional ESSs, leading to the creation of a misspliced message predicted to encode a truncated, non-functional protein. However, these data did not allow us to determine which of the SR protein/hnRNPs motifs were functional. Although it is unlikely that each motif was able to be recognized simultaneously, due to the overlap between them, it is possible that each motif was important in a different cell type, depending on the relative expression levels of SR protein/hnRNPs.

Several putative ESR sequences have been found in exons where they have been sought systematically, raising the possibility of functional redundancy. This may diminish the potential exon-skipping effect of a mutation in any one ESR. However, in cases where 3-10 putative ESE sequences occur within a single exon, a single ESE-disrupting base substitution may lead to efficient exon skipping. Fackenthal et al found that BRCA2 T2722R was a deleterious allele that caused $B R C A 2$ exon 18 skipping (48), and Pagenstecher et al found a silent mutation $M L H 1$ c. $1731 \mathrm{G}>\mathrm{A}$ caused $M L H 1$ exon 15 skipping (54). However, a single ESS-creating base substitution may lead to efficient exon skipping. Oliveira et al found that POMGNT1 c.636C $>\mathrm{T}$ created a new ESS and caused POMGNT1 exon 7 skipping (55), and Raponi et al found that BRCA1 c. $231 \mathrm{G}>\mathrm{T}$ created a new ESS and caused BRCAI exon 6 skipping (56). This suggests that, at least in certain cases, individual ESRs may be critical for splicing even when other ESRs are present in the same exon. However, the splice mutations of $M L H 1$ and $M S H 2$ have been underestimated. The strong correlation between missense mutations with splicing enhancer/silencer motifs found in this study also suggested that splicing-related mutations in the two genes may be relatively common. The computer predictions do not always correlate with in vivo splicing defects. The predictable ESR error mutations require experimental analysis for validation in a further study.

In conclusion, our results indicated that there is a strong correlation between missense mutations in $\mathrm{MLH} 1$ and $\mathrm{MSH} 2$ genes detected in East Asian gastrointestinal cancer patients and ESR motifs. In total, 38 (33.3\%) mutations were predicted to be functionally deleterious and possess one potential ESR error, while $18(15.8 \%)$ mutations were predicted to be functionally deleterious with two potential ESR errors. These may be more likely to affect exon splicing. To truly understand the molecular nature of mutations, splicing patterns should be compared between wild-type and mutant samples.

\section{Acknowledgements}

This study was supported by the National Natural Science Foundation of China (Grant Nos. 81270152, 81070273 and 30972535).

\section{References}

1. Peltomäki P and Vasen HF: Mutations predisposing to hereditary nonpolyposis colorectal cancer: database and results of a collaborative study. The International Collaborative Group on hereditary nonpolyposis colorectal cancer. Gastroenterology 113: 1146-1158, 1997.

2. Lynch HT and de la Chapelle A: Genetic susceptibility to non-polyposis colorectal cancer. J Med Genet 36: 801-818, 1999.

3. Cartegni L, Chew SL and Krainer AR: Listening to silence and understanding nonsense: exonic mutations that affect splicing. Nat Rev Genet 3: 285-298, 2002.

4. Pagani F and Baralle FE: Genomic variants in exons and introns: identifying the splicing spoilers. Nat Rev Genet 5: 389-396, 2004.

5. Shapiro MB and Senapathy P: RNA splice junctions of different classes of eukaryotes: sequence statistics and functional implications in gene expression. Nucleic Acids Res 15: 7155-7174, 1987.

6. Jurica MS and Moore MJ: Pre-mRNA splicing: awash in a sea of proteins. Mol Cell 12: 5-14, 2003.

7. Liu HX, Zhang M and Krainer AR: Identification of functional exonic splicing enhancer motifs recognized by individual SR proteins. Genes Dev 12: 1998-2012, 1998.

8. Blencowe BJ: Exonic splicing enhancers: mechanism of action, diversity and role in human genetic diseases. Trends Biochem Sci 25: 106-110, 2000.

9. Hastings ML and Krainer AR: Pre-mRNA splicing in the new millennium. Curr Opin Cell Biol 13: 302-309, 2001.

10. Zuo P and Maniatis T: The splicing factor U2AF35 mediates critical protein-protein interactions in constitutive and enhancer-dependent splicing. Genes Dev 10: 1356-1368, 1996.

11. Kan JL and Green MR: Pre-mRNA splicing of $\operatorname{IgM}$ exons M1 and M2 isdirected by a juxtaposed splicing enhancer and inhibitor. Genes Dev 13: 462-471, 1999.

12. Cartegni L, Wang J, Zhu Z, Zhang MQ and Krainer AR: ESE finder: a web resource to identify exonic splicing enhancers. Nucleic Acid Research 31: 3568-3571, 2003.

13. Smith PJ, Zhang C, Wang J, Chew SL, Zhang MQ and Krainer AR: An increased specificity score matrix for the prediction of SF2/ ASF-specific exonic splicing enhancers. Hum Mol Genet 15: 2490-2508, 2006.

14. Wang Z, Rolish ME, Yeo G, Tung V, Mawson M and Burge CB: Systematic identification and analysis of exonic splicing silencers. Cell 119: 831-845, 2004.

15. Cai Q, Sun MH, Fu G, et al: Mutation analysis of hMSH2 and hMLH1 genes in Chinese hereditary nonpolyposis colorectal cancer families. Zhonghua Bing Li Xue Za Zhi 32: 323-328, 2003 (In Chinese) 
16. Wang XL, Yuan Y, Zhang SZ, Cai SR, Huang YQ, Jiang Q and Zheng S: Clinical and genetic characteristics of Chinese hereditary nonpolyposis colorectal cancer families. World J Gastroenterol 12: 4074-4077, 2006.

17. Nomura S, Sugano K, Kashiwabara H, et al: Enhanced detection of deleterious and other germline mutations of hMSH2 and hMLH1 in Japanese hereditary nonpolyposis colorectal cancer kindreds. Biochem Biophys Res Commun 271: 120-129, 2000.

18. Kim JC, Kim HC, Roh SA, et al: hMLH1 and hMSH2 mutations in families with familial clustering of gastric cancer and hereditary non-polyposis colorectal cancer. Cancer Detect Prev 25: 503-510, 2001

19. Nakahara M, Yokozaki H, Yasui W, Dohi K and Tahara E: Identification of concurrent germline mutations in hMSH2 and/or hMLH1 in Japanese hereditary nonpolyposis colorectal cancer kindreds. Cancer Epidemiol Biomarkers Prev 6: 1057-1064, 1997.

20. Park SJ, Lee KA, Park TS, Kim NK, Song J, Kim BY and Choi JR: A novel missense MSH2 gene mutation in a patient of a Korean family with hereditary nonpolyposis colorectal cancer. Cancer Genet Cytogenet 182: 136-139, 2008.

21. Fan Y, Liu X, Zhang H, et al: Variations in exon 7 of the MSH2 gene and susceptibility to gastrointestinal cancer in a Chinese population. Cancer Genet Cytogenet 170: 121-128, 2006.

22. Yan HL, Hao LQ, Jin HY, et al: Clinical features and mismatch repair genes analyses of Chinese suspected hereditary non-polyposis colorectal cancer: a cost-effective screening strategy proposal. Cancer Sci 99: 770-780, 2008.

23. Leung SY, Chan TL, Chung LP, et al: Microsatellite instability and mutation of DNA mismatch repair genes in gliomas. Am J Pathol 153: 1181-1188, 1998.

24. Wu MS, Lee CW, Shun CT, et al: Distinct clinicopathologic and genetic profiles in sporadic gastric cancer with different mutator phenotypes. Genes Chromosomes Cancer 27: 403-411, 2000.

25. Kim YM, Choe CG, Cho SK, Jung IH, Chang WY and Cho M: Three novel germline mutations in MLH1 and MSH2 in families with Lynch syndrome living on Jeju island, Korea. BMB Rep 43: 693-697, 2010

26. Zhao C, Tang WZ, Gao F and Li W: Study on hMSH2 gene mutation in the carcinogenesis of sporadic colorectal carcinoma Journal of Guangxi Medical University 27: 11-14, 2010.

27. Tang R, Hsiung C, Wang JY, et al: Germ line MLH1 and MSH2 mutations in Taiwanese Lynch syndrome families: characterization of a founder genomic mutation in the MLH1 gene. Clin Genet 75: 334-345, 2009 .

28. Jin HY, Liu X, Li VK, et al: Detection of mismatch repair gene germline mutation carrier among Chinese population with colorectal cancer. BMC Cancer 8: 44, 2008.

29. Han HJ, Yuan Y, Ku JL, et al: Germline mutations of hMLH1 and hMSH2 genes in Korean hereditary nonpolyposis colorectal cancer. J Natl Cancer Inst 88: 1317-1319, 1996.

30. Sheng JQ, Fu L, Sun ZQ, et al: Mismatch repair gene mutations in Chinese HNPCC patients. Cytogenet Genome Res 122: 22-27, 2008.

31. Cui L, Jin HY, Cheng HY, Yan YD, Meng RG and Yu DH: Genetic detection of Chinese hereditary nonpolyposis colorectal cancer. World J Gastroenterol 10: 209-213, 2004

32. Furukawa T, Konishi F, Shitoh K, Kojima M, Nagai H and Tsukamoto T: Evaluation of screening strategy for detecting hereditary nonpolyposis colorectal carcinoma. Cancer 94: 911-920, 2002

33. Yuen ST, Chan TL, Ho JW, et al: Germline, somatic and epigenetic events underlying mismatch repair deficiency in colorectal and HNPCC-related cancers. Oncogene 21: 7585-7592, 2002.

34. Zhang $\mathrm{CH}, \mathrm{He} \mathrm{YL}$, Wang FJ, et al: Detection of hMSH2 and hMLH1 mutations in Chinese hereditary non-polyposis colorectal cancer kindreds. World J Gastroenterol 14: 298-302, 2008.

35. Zhang Y, Liu X, Fan Y, et al: Germline mutations and polymorphic variants in MMR, E-cadherin and MYH genes associated with familial gastric cancer in Jiangsu of China. Int J Cancer 119: 2592-2596, 2006.

36. Yuan Y, Han HJ, Zheng S and Park JG: Germline mutations of hMLH1 and hMSH2 genes in patients with suspected hereditary nonpolyposis colorectal cancer and sporadic early-onset colorectal cancer. Dis Colon Rectum 41: 434-440, 1998.
37. Yoon SN, Ku JL, Shin YK, et al: Hereditary nonpolyposis colorectal cancer in endometrial cancer patients. Int J Cancer 122: 1077-1081, 2008

38. Sinn DH, Chang DK, Kim YH, et al: Effectiveness of each Bethesda marker in defining microsatellite instability when screening for Lynch syndrome. Hepatogastroenterology 56: 672-676, 2009

39. Shin YK, Heo SC, Shin JH, et al: Germline mutations in MLH1, MSH2 and MSH6 in Korean hereditary non-polyposis colorectal cancer families. Hum Mutat 24: 351, 2004.

40. Zhang XM, Li JT, Zhu M, Wu XL, Gao P, Zhou P and Wang YP: Study on the relationship between genetic polymorphism Val384Asp in hMLH1 gene and the risk of four different carcinomas. Zhonghua Liu Xing Bing Xue Za Zhi 25: 978-981, 2004 (In Chinese).

41. Han HJ, Maruyama M, Baba S, Park JG and Nakamura Y: Genomic structure of human mismatch repair gene, hMLH1, and its mutation analysis in patients with hereditary non-polyposis colorectal cancer (HNPCC). Hum Mol Genet 4: 237-242, 1995.

42. Wang CF, Zhou XY, Sun MH, et al: Two novel germline mutations of MLH1 in hereditary nonpolyposis colorectal cancer family. Zhonghua Bing Li Xue Za Zhi 35: 68-72, 2006 (In Chinese).

43. Sheng JQ, Chan TL, Chan YW, et al: Microsatellite instability and novel mismatch repair gene mutations in northern Chinese population with hereditary non-polyposis colorectal cancer. Chin J Dig Dis 7: 197-205, 2006.

44. Wei W, Liu F, Liu L, et al: Distinct mutations in MLH1 and MSH2 genes in hereditary non-polyposis colorectal cancer (HNPCC) families from China. BMB Rep 44: 317-322, 2011

45. Ng PC and Henikoff S: Predicting deleterious amino acid substitutions. Genome Res 11: 863-874, 2001.

46. Kumar P, Henikoff S and Ng PC: Predicting the effects of coding non-synonymous variants on protein function using the SIFT algorithm. Nat Protoc 4: 1073-1082, 2009.

47. Liu HX, Cartegni L, Zhang MQ and Krainer AR: A mechanism for exon skipping caused by nonsense or missense mutations in BRCA1 and other genes. Nat Genet 27: 55-58, 2001.

48. Fackenthal JD, Cartegni L, Krainer AR and Olopade OI: BRCA2 T2722R is a deleterious allele that causes exon skipping. Am J Hum Genet: 71: 625-631, 2002.

49. Gorlov IP, Gorlova OY, Frazier ML and Amos CI: Missense mutations in hMLH1 and hMSH2 are associated with exonic splicing enhancers. Am J Hum Genet 73: 1157-1161, 2003.

50. Gorlov IP, Gorlova OY, Frazier ML and Amos CI: Missense mutations in cancer suppressor gene TP53 are colocalized with exonic splicing enhancers (ESEs). Mutat Res 554: 175-183, 2004.

51. Nielsen KB, Sørensen S, Cartegni L, et al: Seemingly neutral polymorphic variants may confer immunity to splicing-inactivating mutations: a synonymous SNP in exon 5 of MCAD protects from deleterious mutations in a flanking exonic splicing enhancer. Am J Hum Genet 80: 416-432, 2007.

52. Cartegni L and Krainer AR: Disruption of an SF2/ASF dependent exonic splicing enhancer in SMN2 causes spinal muscular atrophy in the absence of SMN1. Nat Genet 30: 377-384, 2002.

53. Auclair J, Busine MP, Navarro C, et al: Systematic mRNA analysis for the effect of MLH1 and MSH2 missense and silent mutations on aberrant splicing. Hum Mutat 27: 145-154, 2006.

54. Pagenstecher C, Wehner M, Friedl W, et al: Aberrant splicing in MLH1 and MSH2 due to exonic and intronic variants. Hum Genet 119: 9-22, 2006

55. Oliveira J, Soares-Silva I, Fokkema I, et al: Novel synonymous substitution in POMGNT1 promotes exon skipping in a patient with congenital muscular dystrophy. J Hum Genet 53: 565-572, 2008.

56. Raponi M, Kralovicova J, Copson E, et al: Prediction of single-nucleotide substitutions that result in exon skipping: identification of a splicing silencer in BRCA1 exon 6. Hum Mutat 32: 436-444, 2011. 